\title{
Gendergerechtes Forschungsdesign an der Schnittstelle Mensch - Technik
}

\author{
Dorothea Erharter
}

ZIMD - Zentrum für Interaktion, Medien \& soziale Diversität

\section{Zusammenfassung}

Als gender-relevant gelten Forschungen prinzipiell an der Schnittstelle zum Menschen. Forschung im HCI- Bereich ist damit das Kernthema genderrelevanter technologischer Forschung. Im Gegensatz zu Projekten im biomedizinischen Bereich spielen biologische Unterschiede zwischen den Geschlechtern für Mensch-Maschine-Schnittstellen nur eine marginale Rolle. Geschlechtsspezifische Unterschiede müssen daher sehr kritisch betrachtet werden. Statt auf Unterschiede ist der Fokus auf vielfältige Faktoren zu legen, die unterschiedliche Lebensrealitäten und Sozialisationsprozesse berücksichtigen. Anhand von eigenen Forschungsprojekten im Bereich Gender \& Technik zeigt die Autorin auf, wie gendergerechtes Forschungsdesign an der Schnittstelle Mensch zu Technologie konzipiert werden kann. Dieser Artikel versucht aus der Breite an Vorschlägen für gendergerechtes Forschungsdesign die für HCI relevanten und in eigenen Projekten erprobten Methoden herauszufiltern und nachvollziehbar zu machen.

\section{$1 \quad$ Einleitung}

In Materialien zur Berücksichtigung von Gender-Aspekten in Forschungsprojekten ist ein Schlüsselbegriff die Gender-Relevanz. Als gender-relevant gelten alle FTE-Projekte an der Schnittstelle zum Menschen. Human Computer Interaction, oder besser noch Human Technology Interaction ist damit das Kerngebiet genderrelevanter Projekte im IKT-Sektor und in allen technologischen Bereichen. Im biomedizinischen Sektor steht die Physis des Menschen im Zentrum steht und damit können biologische Unterschiede eine wichtige Rolle spielen. Im Bereich des Biomedical Engineering geht es zusätzlich auch um Mensch-Maschine-Schnittstellen, also Fragen der Technologie-Nutzung. In beiden Bereichen wird damit die Kategorie Sex (Geschlecht) ein relevantes Feld für die Forschung und auch überlagernde Effekte von Sex (biologisches Geschlecht) und Gender (soziokulturelles Geschlecht) müssen berücksichtigt werden. Soferne es jedoch ausschließlich um Mensch-Maschine-Schnittstellen geht - also im „Non-Biomedical-Sektor“ -, spielen biologische Unterschiede eine vernachlässigbare Rolle. 
Ein auf geschlechtsspezifische Unterschiede fokussierendes Forschungsdesign birgt daher die Gefahr in sich, Geschlechterstereotypen zu verstärken. Um das zu vermeiden ist der Fokus auf Gender, also beispielsweise auf Unterschiede in den Lebensrealitäten und Sozialisationsprozessen zu legen. Mit diesem Artikel sollen auf Basis des Forschungsstands und der Erfahrungen der Autorin in technologischen Forschungsprojekten die wichtigsten Elemente gendergerechten Forschungs-designs in HCI/HTI zusammengefasst und mit Beispielen belegt werden.

\section{Forschungsstand}

\subsection{Innovationspotential und Gender-Relevanz}

Dass die Berücksichtigung von Gender-Aspekten ein technologisches Innovationspotenzial mit sich bringt, ist mittlerweile gut belegt. Gemäß Bührer \& Schraudner (2006) können dadurch neue Zielgruppen und Nutzungszusammenhänge für technologische Produkte erschlossen werden. Laut Schiebinger können Gender-Aspekte die Entwicklung neuer Produkte und Dienstleistungen bewirken, die den Anforderungen komplexer NutzerInnen-gruppen gerecht werden und damit das menschliche Wohlbefinden einschließlich der Gleichstellung der Geschlechter fördern. Damit werde insgesamt die globale Wettbewerbsfähigkeit und Nachhaltigkeit gefördert. (Schiebinger \& Klinge 2013,116)

Der Begriff der Gender-Relevanz wurde 2006 von Bührer \& Schraudner eingeführt. Als gender-relevant gelten Forschungen und Produktentwicklungen prinzipiell an der Schnittstelle zum Menschen, wobei hier sowohl körperliche als auch soziokulturelle und Nutzungszusammenhänge berücksichtigt werden sollen. (Bührer \& Schraudner 2006).

\subsection{Materialien zu Gender im technologischen Forschungsdesign}

Im Projekt Discover Gender, dessen Federführung beim Fraunhofer Institut für Innovationsforschung lag, wurde ein Leitfaden zur Berücksichtigung von Gender-Aspekten in Forschungs- und Entwicklungsvorhaben entwickelt. Der Leitfaden wurde anhand einer Reihe von Fallbeispielen aus sehr unterschiedlichen technologischen Richtungen überprüft und für NaturwissenschaftlerInnen und TechnikerInnen verständlich dargestellt. (ebenda 2006). Abweichend vom aktuellen Stand der Gender-Forschung wurde in den Beispielen des Leitfadens das Gender-Konzept allerdings auf eine „strikt binäre Logik verengt“. (Bath 2007:2). Er läuft dadurch Gefahr „,Geschlechterstereotype zu verstärken und die Vielfalt der sozialen Welt nur ungenügend zu adressieren. “ (Maaß et al. 2014:68) Die Gender-Studies setzen im Gegensatz dazu eine intersektionale Überlagerung verschiedener Faktoren voraus und fordern beispielsweise eine Berücksichtigung der Lebensrealitäten entlang physischer und soziokultureller Unterscheidungsmerkmale ein.

Das von Londa Schiebinger geleitete, an der University of Stanford initiierte, internationale Projekt Gendered Innovations ist das Leuchtturmprojekt zum Thema. Mehr als 60 ExpertInnen aus ganz Europa, den Vereinigten Staaten und Kanada erarbeiteten von 2009 bis 2012 Materialien zur Integration von Gender-Aspekten in verschiedene naturwissenschaftliche und 
technische Disziplinen. Auf der Plattform http:// http://genderedinnovations.stanford.edu werden die in diesem Projekt erarbeiteten Methoden und inhaltlichen Ergebnisse präsentiert und laufend ergänzt. Sie bauen in grundlegenden Konzepten teilweise auf den Ergebnissen von Discover Gender auf und stehen im Web zur freien Nutzung zur Verfügung (Schiebinger \& Klinge 2013). Seitens der Gender-Studies wird die verwendete Unterscheidung zwischen „,biologischem“ und „sozialem“ Geschlecht kritisiert. (Maaß et al. 2014)

Die große Qualität der beiden Projekte Gendered Innovations und Discover Gender liegt in einer Sammlung von Leitfragen zur Reflexion der Forschungskultur, von Standards und Prämissen der jeweiligen Disziplin, zum Forschungsdesign und zur sprachlichen und visuellen Repräsentation. (Bührer \& Schraudner 2006; Schiebinger \& Klinge 2013)

Maaß et al. stellten mit GERD (Gender Extended Research and Development) ein Vorgehensmodell vor, mit dem Gender- und Diversity-Forschung für die Informatik nutzbar gemacht werden kann. Sie unterscheiden zwischen Kernprozessen, die an ein Wasserfallmodell angelehnt sind, und Reflexionsaspekten, die als Kontext in diese Kernprozesse einfließen. Diese Reflexionsaspekte sind relativ allgemein formuliert und erfordern für die Anwendung zunächst Know-How-Transfer. (Maaß et al. 2014)

\section{Theoretischer Hintergrund}

\subsection{Besonderheiten von HCI/HTI-Projekten}

Nur sehr wenige biologische Unterschiede wirken sich auf Human-Technology-Schnittstellen aus. Diese sind biologisch meist nur mit-beeinflusst und häufig stärker durch Diversity-Faktoren wie das Alter geprägt. Diversity-Faktoren können in HCI-Projekten eine große innovationstreibende Kraft entwickeln (Erharter \& Xharo 2014).

Im Gegensatz zu biomedizinischer Forschung spielen biologische Unterschiede in der IKT und in den Bereichen, in denen es um Mensch-Technologie-Schnittstellen (HTI) geht, fast ausschließlich hinsichtlich Beeinträchtigungen und Behinderungen eine Rolle, wie sie in der Accessibility ( Inclusive Design, Design for All) adressiert werden.

Im Projekt G-U-T konnte vom Team der Autorin festgestellt werden, dass biologische Unterschiede zwischen Frauen und Männern für die Gestaltung von Websites und Apps keine nennenswerte Rolle spielen. Im Projekt MOBISENIORA wurde darüber hinaus bestätigt, dass selbst die Unterscheidung zwischen jung und alt in biologischer Hinsicht auf einige wenige mögliche Beeinträchtigungen hinausläuft, die - zumindest in diesem Projekt - eine weitaus geringere Rolle spielten als vom Team zuvor angenommen. (Erharter et al. 2014)

In den genannten Projekten hat sich gezeigt, dass mit wenigen Ausnahmen, in denen ergonomische Aspekte eine Rolle spielen, in der HCI/HTI-Forschung und -Entwicklung über den Bereich der Accessibility hinausgehende biologische Unterschiede vernachlässigt werden können. Der Umfang der Fragestellungen in Materialien zur Berücksichtigung von Genderund Diversity-Aspekten könnte für diesen Bereich daher deutlich verringert werden. 


\subsection{Geschlechtsspezifische Unterschiede}

Geschlechtsspezifische Unterschiede wurden jahrtausendelang missbraucht um Frauen von gesellschaftspolitischer Beteiligung und Bildung abzuschneiden, und Reste davon existieren heute nach wie vor. Kein Wunder also, dass sich Menschen, die sich thematisch mit dieser Ausgrenzung befassen oder sie sogar am eigenen Leib zu spüren bekommen (haben), die Haare sträuben, wenn mit biologischen Unterschieden argumentiert wird. Eine kritische Auseinandersetzung damit ist daher wesentlich. (vgl. z. B. Hammerl 2009).

Im Gegensatz zu genderspezifischen Unterschieden (soziokulturelle Unterschiede) sind geschlechtsspezifische Unterschiede biologischer Natur. Sie sind meist viel geringer als gemeinhin angenommen. Männer und Frauen liegen in der Ausprägung der meisten Merkmale sehr nahe beisammen. Die Unterschiede innerhalb eines Geschlechts sind (beispielsweise bei der räumlichen Wahrnehmung) viel größer als die Unterschiede zwischen den Geschlechtern. Dennoch wird die Verteilung der Geschlechterunterschiede meist so wahrgenommen, als gäbe es riesige Unterschiede zwischen den Geschlechtern. (Neyer \& Asendorp 2012)

\subsection{Genderspezifische Unterschiede}

In der Geschlechterforschung gibt es den Begriff des Doing Gender. vgl. z. B. Bidwell-Steiner \& Krammer 2010). Damit ist gemeint, dass das soziale Geschlecht im Wesentlichen erst durch das Tun zustande kommt. Verhalte ich mich als Frau, werde ich als Frau wahrgenommen, verhalte ich mich als Mann, werde ich als Mann wahrgenommen. Dies inkludiert Kleidung, Aufmachung, aber Körperhaltung, und auch die Handlungen, Arbeitsteilung etc.

Die Lebensrealitäten der Menschen sind mehr oder weniger stark durch das Doing Gender geprägt, also durch den Vollzug dessen, was das jeweilige Umfeld von Männern bzw. Frauen erwartet. Die Lebensrealitäten können sich in vielfältiger Weise unterscheiden, hier spielen auch andere Diversity-Faktoren eine Rolle. Wichtig ist, sich bewusst zu machen, dass Unterschiede zwischen Männern und Frauen viel mehr als durch biologische Unterschiede durch das Doing Gender zustande kommen. (ebenda)

Beispielsweise wirkt es sich massiv auf Nutzungsszenarien aus, ob jemand Betreuungspflichten hat, da diese Personen im Laufe eines Tages deutlich mehr Wege zurück legen, stärker abgelenkt sind, etc. Dass sie dadurch andere Anforderungen, zum Beispiel an Navigationssysteme, haben können, ist nicht an das biologische Geschlecht gekoppelt, es ist eine GenderThematik. (vgl. Scambor \& Zimmer, 2012). In der HCI/HTI-Forschung ist daher nicht nur von Männern und Frauen auszugehen, sondern von Menschen mit vielen verschiedenen Merkmalen und in vielen verschiedenen Nutzungskontexten, da sonst die Gefahr der Stereotypisierung besteht. Davon profitieren auch Männer, wie Raewyn Connell gezeigt hat (2006), die von verschiedenen "Männlichkeiten" spricht. 


\section{Gendergerechtes Forschungsdesign}

\subsection{Projekte}

Die Autorin hat in den letzten Jahren im ZIMD einige technologische Forschungsprojekte mit Gender-Fokus im Kontext von Mensch-IKT-Schnittstellen durchgeführt, die als Basis für diesen Artikel dienen. In all diesen Projekten befindet sich gendergerechtes Forschungsdesign immer im Spannungsfeld von geschlechtsspezifischen Unterschieden und der Kritik daran bzw. dem Versuch gender(!)spezifische Unterschiede zu berücksichtigen. Dies hat damit zu tun, dass es beim Versuch Gender zu berücksichtigen, ja vordergründig um Unterschiede zwischen Männern und Frauen geht. Und da Männlichkeit und Weiblichkeit auf den ersten Blick biologisch bedingt scheinen ${ }^{1}$, wird zunächst fast immer auf biologische Unterschiede fokussiert.

Im Projekt $G$ - $U$ - $T$ (http://g-u-t.zimd.at) haben wir durch vergleichende Analyse überprüft, welche Gender-Aspekte und Diversity-Dimensionen in der Praxis für das Design und Development von Apps und Websites relevant sind, und eine Guideline zu deren Berücksichtigung entwickelt. (Erharter 2014-2). Im Projekt MOBISENIORA (www.mobiseniora.at) wurde die Nutzung von Smartphones und Tablets durch Seniorinnen und Senioren unter Berücksichtigung von Gender-Aspekten untersucht. Derzeit werden Leitfäden für App-Entwicklung, Bildungsangebote sowie Verkaufsberatung und Support durch Telekom-Anbieter entwickelt. (Amann et al. 2015). Im Projekt GEMPLAY (www.gemplay.at) werden gendergerechte Spielkonzepte zur Bewegungsförderung entwickelt. Bislang wurde eine Online-Studie abgeschlossen, derzeit werden Usability-Tests mit Prototypen durchgeführt. Im Projekt GENSISYS wird ein Methodenset zur Evaluation von Gender und Diversity Dimensionen für Ergonomie und Usability an Arbeitsplätzen in sicherheitskritischen Systemen erarbeitet. (Erharter 2014-1). Ein Teil der Projektergebnisse ist bereits publiziert und wurde - auch methodisch - im Rahmen internationaler Konferenzen diskutiert. Im Folgenden fasse ich die methodischen Erkenntnisse zu gendergerechtem Forschungsdesign aus diesen Forschungsprojekten zusammen und belege sie mit Beispielen.

\subsection{Literaturbetrachtung}

Ein wichtiger Ausgangspunkt jeglicher Forschungsprojekte ist die vorhandene Literatur. Eine Fülle von Studien scheinen geschlechtsspezifische Unterschiede zwischen Frauen und Männern zu belegen. Auf den ersten Blick erscheinen jedoch häufig Unterschiede als biologisch, die in Wirklichkeit vor allem auf Lernerfahrungen, also auf soziokulturellen Unterschieden beruhen. Solche scheinbar biologischen Unterschiede kommen häufig durch ein unsauberes Forschungsdesign zustande, und bilden dann eher die Zuschreibungen und Vorannahmen der Forschenden ab, als real existierende Unterschiede. (vgl. Precht 2010). Es kann davon ausgegangen werden, dass es sich bei den meisten dokumentierten geschlechtsspezifischen Unterschieden vor allem im Bereich der Evolutionsbiologie entweder um erlernte oder um solch

1 Zum „Schein“ des biologischen Geschlechts siehe zum Beispiel Engel 2002. 
scheinbare Unterschiede handelt. Darüber hinaus hat sich gezeigt, dass Studien, die Unterschiede zwischen Frauen und Männern belegen, sehr viel häufiger zitiert werden, als Studien, die belegen, dass es keine Unterschiede gibt (vgl. Fine, 2012).

Eine günstige Vorgangsweise um geschlechtsspezifische Unterschiede in der Literatur einer kritischen Betrachtung zu unterziehen, besteht zum einen in der Schärfung eines kritischen Blicks: Wie wurden solche Studien gemacht? Sind sie prinzipiell in sich sauber gemacht? Mit welcher Brille wurde das Forschungsdesign gemacht? Welche Sprache wird verwendet? Welche Vorannahmen spiegeln sich in den Ergebnissen? Zum anderen in der Recherche kritischer Perception: Wie werden die Quellen in der Literatur diskutiert? Was wird kritisiert und von wem? Was wird zitiert, und von wem?

Ein wichtiger Aspekt betrifft auch die Wiedergabe von Forschungsergebnissen. In einem Vortrag auf der Gender-UseIT hat die Autorin den Begriff „Forschungs-Stille-Post“ geprägt und meint damit den Vorgang der Reduktion differenzierter Ergebnisse auf Verallgemeinerungen, wie „Frauen haben Schulterbeschwerden“. (Erharter 2014-1).

Im Projekt GENSISYS wurden zahlreiche für HCI-Design relevante Studien gesichtet, die geschlechtsspezifische Unterschiede reflektieren. Die meisten Unterschiede waren entweder recht dünn belegt oder durch soziokulturelle Faktoren zu erklären. Bewährt hat sich in diesem Projekt, aus den in der Literatur gefundenen Unterschieden Bereiche zu identifizieren, in denen es prinzipiell größere Unterschiede geben kann, als bisher vielleicht berücksichtigt, unabhängig davon, welche Dimensionen (Alter, Geschlecht, Kultureller Hintergrund etc.) zu diesen Unterschieden führen.

\subsection{Hypothesen bzw. Forschungsfragen}

Fokussiert das Forschungsdesign auf geschlechtsspezifische Unterschiede, wird auf diese ein Vergrößerungsglas gerichtet: Sie erscheinen größer als sie tatsächlich sind. Damit reproduzieren sie Ungleichheiten, die zusammengenommen zu gesellschaftlichen Ungleichwertigkeiten führen können. Für HCI-Forschung relevante Unterschiede können darüber hinaus sehr vielfältige Ursachen haben.

Für die Formulierung von Hypothesen in der anwendungsorientierten technologischen Forschung bedeutet das, den Fokus auf den Bereich der festgestellten Unterschiede zu richten und die Frage, wodurch diese Unterschiede verursacht sind, erst in zweiter oder dritter Linie zu stellen.

Im Projekt GENSISYS beispielsweise wurden Hypothesen, die in der Einreichphase geschlechtsspezifisch formuliert waren (A), vom uns folgendermaßen zu Forschungsfragen umformuliert (B):

A. Räumliche Wahrnehmung: Durch größere Bildschirme kann die räumliche Wahrnehmung zugunsten der Frau im Vergleich zum Mann verbessert werden.

B. Es gibt Studien, die zeigen, dass die räumliche Wahrnehmung von Frauen durch größere Bildschirme verbessert mehr werden kann als von Männern. Wie wirken sich größere Bildschirme in der konkreten Situation aus? 
Größere Bildschirme können für verschiedene Personen Vor- und/oder Nachteile haben, aufgrund verschiedener Faktoren, von denen einer das Geschlecht sein kann. Wichtig ist, die Inhalte vom biologischen Geschlecht zu lösen. Die Fragestellung wird dadurch auch offener für weitere Faktoren.

An diesem Beispiel zeichnet sich darüber hinaus ein weiteres Merkmal gendergerechter Forschung ab: die Formulierung qualitativer Fragestellungen. Mit quantitativen Fragestellungen kann überwiegend auf vordefinierte Kategorien und Merkmale eingegangen werden, die per se dazu angetan sind, Vorannahmen zu bestätigen. Qualitative Fragestellungen sind zwar aufwändiger auszuwerten, aber ergebnisoffener und daher prinzipiell besser geeignet für ein gendergerechtes Forschungsdesign (siehe Kap. 4.5).

\subsection{Quantitative Studien: Clustern, aber wie?}

Ein wesentliches Merkmal quantitativer Studien sind Cluster, die gebildet werden, um Aussagen über bestimmte Gruppen treffen zu können. Angesichts der Gefahr der Verstärkung von Stereotypen empfiehlt es sich, nicht entlang vorgegebener Merkmale wie (Geschlecht/Alter/...) zu clustern, sondern ergebnisoffen entlang von Faktoren, die sich aus der Studie selbst oder aus zuvor gemachten qualitativen Methoden ergeben. Damit kann die Fortschreibung stereotyper Zuschreibungen vermieden werden.

Innerhalb dieser Cluster soll dann die Geschlechterverteilung betrachtet werden. Dies ist zentral, da ohne diesen Schritt die Kategorie „Gender“ aus dem Blick geraten würde. Vorhandene Ungleichheiten würden dadurch unsichtbar gemacht. Wolffram \& Winker (2005) haben beispielsweise in einer Studie über technische StudienanfängerInnen zunächst nach Technikhaltungen geclustert, und erst danach die Geschlechterverteilungen innerhalb der fünf Gruppen betrachtet. Mit einer solchen Vorgangsweise fällt es viel leichter, der Falle vorschneller Zuschreibungen zu entgehen.

Ist dies nicht möglich, und müssen Cluster auf Basis vorher festgelegter Merkmale gebildet werden, so empfiehlt es sich Merkmale zu wählen, die weniger durch Stereotypien geprägt sind. Die inneren Persönlichkeitsmerkmale: Geschlecht, Alter, soziale Herkunft, Ethnie, geistige/körperliche Fähigkeiten/Einschränkungen, sexuelle Orientierung (Gardenswartz \& Rowe 2002) und zusätzlich auch Religion/Weltanschauung sind häufiger mit Vorannahmen verknüpft und damit sind stereotype Zuschreibungen (die es immer gibt) schwerer erkennbar.

Im Projekt GEMPLAY wird beispielsweise nach „Spielertypen“ geclustert, innerhalb derer dann die Geschlechter- und Altersverteilung betrachtet wird. Im Projekt MOBISENIORA konnten Gruppen nach Nutzungsverhalten gebildet werden, weitere Faktoren waren Regionalität und jahreszeitliche Einflüsse.

Eine gute Möglichkeit besteht darin, bereits in das Design einer quantitativen Befragung mögliche Auswirkungen von Lebensrealitäten einfließen zu lassen. So wird im Projekt GEMPLAY beispielsweise nach Zeitverwendung und Freizeitverhalten gefragt. Dies kann allerdings qualitative Befragungen nicht ersetzen. 


\subsection{Qualitative Studien: Die Frage nach den Lebensrealitäten}

Wie gezeigt wurde, betreffen für HCI/HTI-Projekte relevante Gender-Aspekte vor allem die unterschiedlichen Lebensrealitäten und Einstellungen von Männern und Frauen. Hier sind insbesondere die folgenden Bereiche relevant:

- Raum-zeitliche Rahmenbedingungen und Wege, die wesentlich von den Lebensumständen und eventuellen Betreuungspflichten geprägt sind (vgl. Scambor \& Zimmer 2012)

- Werthaltungen und Einstellungen gegenüber Technik, die wesentlich durch Sozialisationsprozesse geprägt sind (vgl. Amann-Hechenberger 2015, Kap. 5)

- Technikerfahrung und Technikwissen, die wesentlich durch die berufliche Biographie geprägt sind. (vgl. Erharter 2008)

Um diese Einflussgrößen strukturiert zu erfassen, muss offen gefragt und die Nähe zu den NutzerInnen gesucht werden. Partizipative Methoden sind dafür unerlässlich. Egal, welche Methoden aus dem vielfältigen Vokabular des Partizipatory Design gewählt werden: Die Fragestellungen sollten die oben genannten Bereiche abdecken bzw. dafür offen gehalten und in der Auswertung auf diesbezügliche Unterschiede geachtet werden.

Im Projekt MOBISENIORA wurden beispielsweise mittels semistrukturierter Interviews die Technikhaltungen und das Nutzungsverhalten von SeniorInnen und ihre Erwartungen an Smartphones/Tablets im Kontext ihrer Sozialisationsprozesse betrachtet. Dabei konnten sehr vielfältige Aspekte einfließen. In der Auswertung konnten einige gender(!)spezifische Unterschiede sichtbar gemacht werden. Im Projekt GEMPLAY fließen die unterschiedlichen Lebensläufe, die bei Frauen oft deutlich vielfältigere Aktivitäten beinhalten, ein, indem Participatory Design Workshops gesondert mit rein weiblichen und rein männlichen Gruppen gehalten werden. Im Projekt GENSISYS kamen die aufschlussreichsten und spannendsten Ergebnisse durch qualitative Methoden wie Kontextanalyse, Arbeitsplatzbeschreibung und Tagebuch zustande, auch wenn sich darin kaum genderspezifische Unterschiede spiegelten.

\subsection{Auswahl der Testpersonen}

Zentral für die Berücksichtigung von Gender-Aspekten in partizipativen Methoden ist die Auswahl der Testpersonen. Mindestens ebenso wichtig wie eine ausgewogene Zusammensetzung nach biologischem Geschlecht ist die Berücksichtigung der drei oben genannten Merkmale raumzeitliche Rahmenbedingungen, Technikhaltungen sowie Technikaffinität und -erfahrung als Auswahlkriterien für Testpersonen bzw. als Auswertungskriterien (z. B. viel/mittel/wenig Technikerfahrung). Darüber hinaus kommt hier wieder die Diversity ins Spiel, und es muss betrachtet werden, ob insbesondere bei den inneren Persönlichkeitsmerkmalen (vgl. Kap. 4.3) für das Projekt relevante Kriterien existieren.

Im Projekt MOBISENIORA beispielsweise wurden die Testpersonen entlang einer Matrix ausgewählt, in der neben Geschlecht auch Alter, Regionalität und Nutzungserfahrung berücksichtigt war. Im begleitenden Fragebogen zu den Usability-Tests wurden die Testpersonen dann nach der wöchentlichen Gerätenutzung in Stunden gefragt. In der Auswertung wurde der 
Median der jeweiligen Gerätenutzung gebildet und daraus dann die Gruppen gebildet (viel/wenig/keine Gerätenutzung), für die nach Korrelationen zu anderen Ergebnissen gesucht wurde.

\section{$5 \quad$ Fazit}

Die Vorschläge der AutorInnen für ein gendergerechtes Forschungsdesign im HCI/HTI-Bereich betreffen

- eine kritische Betrachtung von Literatur die zu geschlechtsspezifischen Ergebnissen kommt;

- eine genaue Sprache bei der Wiedergabe geschlechtsspezifischer Forschungsergebnisse;

- eine qualitative Formulierung von Forschungsfragen;

- für quantitative Studien eine Vorgehensweise zu Clusterung und Auswertung;

- für qualitative Studien die Ausschöpfung des Potenzials partizipativer Forschungs- und Designmethoden

- die Blickrichtung auf raum-zeitliche Rahmenbedingungen und Lebensrealitäten (z.B. Betreuungspflichten); auf Werthaltungen, Technikhaltungen und Sozialisationsprozesse; und auf Technikerfahrung und -wissen;

- die Auswahl von Testpersonen;

- das explizite Beschreiben von nicht-vorhandenen Unterschieden, wenn keine gefunden wurden.

Wichtig ist, diese Vorschläge zu gendergerechtem Forschungsdesign in HTI-Projekten nicht als Einzelmaßnahmen, sondern als Paket zu betrachten und anzuwenden. Natürlich kann schon die Anwendung einer einzelnen Maßnahme wichtig uns sinnvoll sein, doch erst im Paket können die Vorschläge ihre volle Innovationskraft entfalten.

\section{Literaturverzeichnis}

Amann-Hechenberger, B., Buchegger, B., Erharter, D., Felmer, V., Fitz, B., Jungwirth, B., Kettinger, M., Schwarz, S., Knoll, B., Schwaninger, T. \& Xharo, E. (2015). Tablet \& Smartphone: Seniorinnen und Senioren in der mobilen digitalen Welt. Forschungsbericht zum Projekt „,mobi.senior.A “. Wien.

Adamo-Villani, N., Wilbur, R. \& Wasburn, M. (2008). Gender Differences in Usability and Enjoyment of VR Educational Games. In: Visualisation, 2008 International Conference. S. 114-119.

Connell, R. (1999). Der gemachte Mann: Konstruktion und Krise von Männlichkeiten. Berlin: Springer.

Bath, C. (2007). Discover Gender in Forschung und Technologieentwicklung. Soziale Technik 4/2007. www.sts.aau.at/ias/content/download/1656/7774. Zuletzt abgerufen 5.10.2014.

Bidwell-Steiner, M. \& Krammer, S. (2010). (Un)Doing Gender als gelebtes Unterrichtsprinzip: Sprache - Politik-Performanz. Wien.

Bührer, S., \& Schraudner, M. (Eds.) (2006). Wie können Gender-Aspekte in Forschungsvorhaben erkannt und bewertet werden? Karlsruhe: Fraunhofer Verlag.

Engel, A. (2002). Wider die Eindeutigkeit. Sexualität und Geschlecht im Fokus queerer Politik der Repräsentation, Frankfurt/M.: Campus. 
Erharter, D. (2008). Bildschirmbasierte Applikationen für ältere User. In: Edith Maier \& Pascale Roux (Hrsg.): UDay VI. Seniorengerechte Schnittstellen zur Technik. Zusammenfassung der Beiträge zum Usability Day VI.

Erharter, D. \& Xharo, E. (2014). Gendability. Gender \& Diversity bewirken innovative Produkte. In: Marsden, N. \& Kempf, U. (Hrsg.): Gender-UseIT 2014 (\#GUI2014). HCI, Web-Usability und UX unter Gendergesichtspunkten. S. 127-141.

Erharter, D. (2014-1). GenSiSys - Gendergerechte Gestaltung von Arbeitsplätzen im Bereich sicherheitskritischer Systeme. Vortrag im Rahmen der Konferenz Gender Use IT, 3.-4. April 2014. Berlin.

Erharter, D. (2014-2). Gender- und Diversity-Faktoren in interaktiven Medien. In: Markus Seidl \& Grischa Schmiedl (Hrsg.): Forum Medientechnik - Next Generation, New Ideas. Glückstadt.

Erharter, D., Jungwirth, B., Knoll, B., Schwarz, S., Posch, P. \& Xharo, E. (2014). Smartphones, Tablets, App für Seniorinnen und Senioren. In: Kemper, Guido (Hrsg.): Assistenztechnik für betreutes Wohnen. AAL Testregion Westösterreich. Tagungsband zum uDay XII. S. 221-235

Fine, Cordelia (2012). Die Geschlechterlüge. Stuttgart: Klett-Cotta Verlag.

Gardenswartz, L. \& Rowe, A. (2002). Diverse Teams at Work. Society for Human Resource Management. 2002.

Gaver, B., Dunne, T. \&; Pacenti, E. (1999). Cultural Probes. Magazine Interactions vol. 6. S. 21-29.

Hammerl, E. (2009). Was ist Gender? Geschlechtsspezifische Rollenzuweisungen und ihre alltäglichen Auswirkungen. In: Hochleithner, M. (Hg): Gender Medicine. Ringvorlesung an der Medizinischen Universität Innsbruck. Band 2. Wien. S. 9-17.

Joost, G. \& Buchmüller, S. (2009). Women's Phone - a Case Study on Knowledge Management in a Participatory Design Process. IASDR 09 Conference. Seoul/ Korea, 18th to 22th October 2009

Maaß, S., Draude, C. \& Wajda, K. (2014). Das GERD-Modell. In. Marsden, N. \& Kempf, U. (Hrsg.): Gender-UseIT - HCI, Web-Usability und User Experience unter Gendergesichtspunkten. Oldenbourg. S. 127-141

Neyer, F. J. \& Asendorpf, J. B. (2012). Geschlechtsunterschiede. Grafik abgerufen von http://link.springer.com/chapter/10.1007/978-3-642-30264-0_7\#page-1 am 26.02.2014.

Precht, R. D. (2010). Liebe. Ein unordentliches Gefühl. München.

Scambor, E. \& Zimmer, F. (2012). Die intersektionelle Stadt. Bielefeld: transcript.

Schiebinger, L. \& Klinge, I. (2013). Gendered Innovations. How Gender Analysis Contributes to Research. Report oft he Expert Group „Innovation through Gender“. European Commission.

Wolffram, A. \& Winker, G. (205). Technikhaltungen von Studienanfängerinnen und -anfängern in technischen Studiengängen. Auswertungsbericht der Erstsemesterbefragung an der TUHH im WS 03/04.

Xharo, E. \& Erharter, D. (2014). SeniorInnen und Usability bei Smartphones und Tablets. In: Seidl, M. \& Schmiedl, G. (Hg.). Forum Medientechnik - Next Generation, New Ideas. Glückstadt. S. 255-268.

\section{Kontaktinformationen}

Dorothea Erharter, ZIMD, 1180 Wien, Währinger Straße 81/12.

d.e@zimd.at.+43-699-11369902. 